\title{
Clustering of protons as the main factor in increasing the activity of leaching solutions at their electrochemical and photochemical processing
}

\author{
Arthur Sekisov* 1,2 and Anna Rasskazova ${ }^{1}$ \\ ${ }^{1}$ Mining Institute FEB RAS, 51 Turgenev st., Khabarovsk, 680000, Russia; \\ ${ }^{2}$ Transbaikal State University, 672039, Chita, Russia
}

\begin{abstract}
The theoretical substantiation for the proton clustering process during electrochemical and photochemical processing of technological solutions is provided in the paper. This process provides a significant increase in the efficiency of leaching of gold from refractory ores. It was proposed that the chemical processes in aqueous solutions occur not only due to electronic (interelectronic) interactions, but also as a result of proton-electron interactions. The hypothesis is substantiated. Protonelectron interactions play an important role in the destruction and formation of chemical bonds between the solvent and the solute, as well as between dissolved substances. Compounds with a polymer-like structure can be formed during the electrolysis of aqueous solutions of different reagents, containing oxygen dimers (O-O). Hydrated cluster shell of active water molecules is formed around these polymer-like compounds. A metastable region of clustered protons is formed. Water-gas emulsion is formed during electrolysis and irradiatin with UV light. Highly active oxidants such as atomic oxygen, superoxide radical ion, ozone, and hydroxyl radical are synthesized in the gas bubbles. These compounds intensify protonation processes during hydration. . The results of experiments on column activation leaching of dispersed gold from largevolume sample of refractory ore from the Pogromnoye deposit prove the validity of the theoretical substantiations.

Key words: protons, clusters, clustering of water, process solutions, electrochemical treatment, photochemical treatment, initiated dissociation of water, reactive oxygen species, percolation leaching, refractory ores, intensification of leaching.
\end{abstract}

\section{Introduction}

The development of the mineral resource base of precious metals is mainly associated with the large-scale processing of ores with dispersed mode of occurrence in the crystal lattice of host (associated) minerals.

\footnotetext{
* Corresponding author: sekisovag@mail.ru
} 
The recovery of such mode of occurrence of precious metals from mineral raw material requires the use of complex multistage mineral processing and hydrometallurgical schemes. Currently, significant progress has been made in the processing of high-grade ores and concentrates containing dispersed forms of precious metals. The progress became possible due to the use of effective processes of bacterial and autoclave oxidation of sulfide and sulfosalt minerals and carbonaceous matter. At the same time, due to the depletion of the mineral resource base of precious metals, namely the development of deposits that are amenable to preliminary concentration of high-grade ores, with relatively simple mining conditions, it is objectively required to develop technologies for extracting dispersed forms of gold and platinum group metals from low-grade refractory ores and technogenically transformed mineral raw materials.

The development of such technologies can be carried out on the basis of studies of the physicochemical features of the cluster forms of occurrence of precious metals in a mineral substance; the search for effective methods of microstructural transformation of the crystal lattices of host minerals. Transformations in associated minerals is accompanied both with the formation of a system of additional microdefects and relocation and/or defragmentation of precious metal clusters. Chemogenic defect formation of crystal lattices of mineral concentrators of precious metals and the relocation or transformation of their clusters can be provided by the use of agents such as collective protons. Collective protons intensively react with the electronic shells of mineral-forming atoms.

\section{Objects, materials and research methods}

The objects of research are the processes of formation of active clustered sub-molecular structures and clustered protons in water and aqueous technological solutions. Materials for research were aqueous solutions of leaching reagents, gold-bearing mineral raw materials. Research methods is the analysis and generalization of research data on clustering processes in a liquid medium, theoretical substantiation of clustering processes in water and aqueous solutions during their electrochemical and photochemical processing, experimental studies of the processes of leaching dispersed gold from refractory ores with electro-photoactivated solutions.

\section{Theoretical substantiation of the activation processes of technological solutions for leaching of refractory gold}

Hydroxyl ions $\mathrm{OH}^{-}$and protons $\left(\mathrm{p}^{+}\right)$are formed in aqueous solutions with neutral $\mathrm{pH}$ and even in distilled water, due to the fluctuation dissociation of its molecules. Protons, almost immediately, bond one of the nearest water molecules and form hydronium ions $\left(\mathrm{H}_{3} \mathrm{O}^{+}\right)$, but upon contact with electrons of a neighboring water molecule or soluble substances, the formed hydronium ion quickly dissociates to a secondary proton and a neutral molecule. At the moment of implantation of a proton into the electron shell of the nearest water molecule, its structural and energy parameters are obviously changed. It, in turn, leads to bond rupture or formation of intermolecular chemical bonds with its neighboring molecules of both water and solutes. In the first case, water clusters are formed due to the compaction of the coupled electron shells; in the second, the dissociation of dissolved substances and/or a chemical reaction between them occurs. Therefore, in aqueous solutions, chemical processes occur not only due to electronic (interelectronic) interactions, but also as a result of proton-electronic interactions, which play an important role in the destruction and formation of chemical bonds between interacting substances. According to modern concepts, even a covalent bond between some substances can be formed by the donor- 
acceptor mechanism, namely, through the attachment of a proton of one of them to the electron shell of one of the atoms of the other, which has a lone pair of electrons. This process is most typical for hydrogen chloride, which actively dissociates in water, and the resulting proton is accepted by the nitrogen atom that is part of the methylamine molecule [1]:

$$
\mathrm{CH}_{3} \mathrm{NH}_{2}+\mathrm{HCl}=\left(\mathrm{CH}_{3} \mathrm{NH}_{3}\right)^{+} * \mathrm{Cl}^{-} \text {. }
$$

From the position of quantum chemistry, the lone-electron pair of the nitrogen atom participates in the formation of bond with hydrogen, and the proton provides its vacant orbital for it, acting as an acceptor. The nitrogen atom, being an electron donor for the hydrogen ion, gets a positive charge. In fact, due to the protonation of the nitrogen atom, the electronic shell of the entire methylamine molecule is changed and its metastable chemical bond with the periodically ionized chlorine atom is formed.

In a more general case, when the protons, formed during the dissociation of water molecules, penetrate into the electronic shells of atoms or ions of solutes, they can perform an intermediate function, i.e. temporarily bond the electronic shells of the solvent molecules (ions) and the atoms (molecules) of the solute. For dissolved substances with a relatively simple structure of the outer electron shells (alkali metals, halogens), the penetration of even individual protons into them causes significant changes in its structural and energy parameters, and, accordingly, chemical activity. At the same time, for substances whose atoms have a complex structure of outer shells, especially with hybrid (s-p, p-d sublevels), its change during protonation becomes possible only when interacting with several protons at once. To transfer into the liquid phase such complexly organized structures as clusters of rare and noble metals, collective protons must also have sufficient energy to significantly change the structural and energy parameters of their electron shells. Therefore, to increase the intensity and completeness of the leaching of transition metals and, in particular, gold from ores, active clustered protons must be present in the process solution. It is obvious that the number of active protons in water clusters depends primarily on the number of its protonated molecules and their energy, which, in turn, depends on the concentration of clustering agents. It is known that the agents (or centers) of clustering of water molecules are radical or ion-radical forms of oxygen and its superperoxide compounds with hydrogen: hydroxyl radicals and other oxygen-containing superperoxide compounds. An increase in the concentration of such compounds leads to an increase in the probability of the formation of multinuclear water clusters in water, respectively, to an increase in the number of clustered protons in their external collective electron shell.

The formation of ionized and radical reaction-active centers of water clustering can be provided by various methods of processing aqueous solutions of the initial reagents, but the most acceptable of them for practical use are electrolysis, photolysis, and their combination [2-3]. During the electrolysis of solutions of a number of salts and acids, the dissociation of water molecules is significantly intensified. It increases the energy of the hydration shells of aqua ions. Compounds with a polymer structure and/or with an increased oxygen content can be obtained as a result of electrolysis. These compounds after their hydration form high-energy clusters of water. In particular, during the electrolysis of a solution of sulfuric acid $\left(\mathrm{H}_{2} \mathrm{SO}_{4}\right)$, peroxymonosulfuric and persulphuric acids $\left(\mathrm{H}_{2} \mathrm{SO}_{5}, \mathrm{H}_{2} \mathrm{~S}_{2} \mathrm{O}_{8}\right)$ [4] are formed in the anode zone; electrolysis of nitric acid forms pernitric $\left(\mathrm{HNO}_{4}\right)$ acid and electrolysis of sodium bicarbonate $\left(\mathrm{NaHCO}_{3}\right)$ produces percarbonic acids $\left(\mathrm{H}_{2} \mathrm{CO}_{4}, \mathrm{H}_{2} \mathrm{C}_{2} \mathrm{O}_{6}\right)$ in the anode zone, and alkali metal superoxides $\left(\mathrm{Na}_{2} \mathrm{C}_{2} \mathrm{O}_{6}, \mathrm{~K}_{2} \mathrm{C}_{2} \mathrm{O}_{6}\right)$ in the interelectrode space. During the electrolysis of sodium chloride solution, hypochlorous $(\mathrm{HClO})$ and perhypochlorous acid $\left(\mathrm{H}_{2} \mathrm{Cl}_{4} \mathrm{O}_{4}\right)$ are formed in the anode zone; sodium hypochlorite $(\mathrm{NaClO})$ in the interelectrode space. Thus, the electrolysis of aqueous solutions of the different reagents can form compounds with a conditionally polymer structure containing a protonated dimer ("bridge") O-O. A hydration cluster shell of active water molecules is formed around $\mathrm{O}-\mathrm{O}$ 
bond. Accordingly, in this case, a metastable region of clustered protons is formed in such an active hydration shell. When a water-gas emulsion formed during electrolysis is irradiated with UV light, highly active oxidants such as atomic oxygen, superoxide radical ion $\left(\mathrm{O}^{*-}\right)$, ozone, and hydroxyl radical $\left(\mathrm{OH}^{*}\right)$ are formed in the volume of gas bubbles. The energy of such water-hydroxyl clusters $\mathrm{OH}^{*}\left(\mathrm{H}_{2} \mathrm{O}\right)_{n}$ (where $\left.\mathrm{n}=2-30\right)$, and, accordingly, its reactivity is much higher than that of ordinary water clusters that form around its ionic dissociates $\left(\mathrm{H}^{+}, \mathrm{OH}^{-}\right)$, for example $\mathrm{H}^{+}\left(\mathrm{H}_{2} \mathrm{O}\right)_{n}$. The redox potential of the $\mathrm{OH} *$ hydroxyl radical reaches $2.8 \mathrm{~V}$ [5], while that for atomic oxygen is $2.42 \mathrm{~V}$, and ozone is $2.07 \mathrm{~V}$. From this fact it follows that both uncoupled electron of an oxygen atom, and, to a greater extent, an active hydrogen atom or formed during internal dissociation of a hydroxyl radical by an ionic "mechanism" a proton changes the structural and energy parameters of the electron shells of water molecules as a result of contact (penetration). Since the system consisting of excited oxygen and hydrogen atoms is metastable, it can be assumed that the electron of the hydrogen atom periodically transfers to the free orbit of the electron shell of the oxygen atom. In this case, such a radical carries out a periodic "internal" dissociation $\mathrm{OH}^{*}=\left(\mathrm{O}^{*} * \mathrm{H}^{+}\right)$. In fact, a positively charged hydrogen ion in such a system is a proton with a sufficiently high energy; accordingly, there is a possibility that its location is not limited only by the electron shell of the oxygen radical ion associated with it. Therefore, the system $\left(\mathrm{O}^{*-*} \mathrm{H}^{+}\right)$can be considered as a relatively weakly bound ion-radical pair. This system, on the one hand, has the function of an acceptor of protons, on the other hand, an acceptor of electrons. The oxygen radical ion $\mathrm{O}^{*-}$ polarizes and attracts with its densified electron shell one of the hydrogen atoms of the neighboring water molecule. The couple $(\mathrm{O}$

${ }^{*} * \mathrm{H}^{+}$) is restored and the cycle repeats with the involvement of other neighboring water molecules. The primary proton $\mathrm{p}^{+}$, separated from the $\mathrm{OH}^{*}$ radical, penetrates into the electron shell of the hydroxyl ion formed during the dissociation of the nearest water molecule. In turn, this leads to the initiation of dissociation of water molecules surrounding the hydroxyl radical with the formation of a system of clustered protons and hydroxyl ions. Due to the greater mobility of protons (relative to hydroxyl ions), they, penetrating into the central parts of the collected electron shells of water molecules of the next layer, compact them, which determines the process of clustering of water.

Highly active radical ions and radicals, first of all, $\mathrm{O}^{*-}$ and $\mathrm{OH}^{*}$ create clusters when interacting with film water surrounding gas bubbles. These clusters are characterized with a limited number of protons in their outer shell, but characterized with high energy. Further clustering of water molecules occurs by combining multinuclear clusters formed during electrolysis and highly active mononuclear clusters formed around "inoculating" $\mathrm{OH}^{*}$ radicals or oxygen radical ions $\left(\mathrm{O}^{*}\right)$ [6].

When complexing agents dissolve in such an active aqueous medium, proton-electron clusters presented in their hydration shells change the character and energy of the collective electron shells of precious metal clusters in a mineral medium. It provides the possibility of transfer of precious metal clusters into pore and microcrack solutions. In this case, prior to the formation of complex anions of gold $\left[\mathrm{Au}(\mathrm{CN})_{2}\right]^{-}$, clustered gold hydrides $\mathrm{Au}_{2} \mathrm{H}_{2}$ or complex compounds $\mathrm{Au}(\mathrm{HCN})_{2}$ are formed. These compounds are transformed into an ion pair $\mathrm{AuH}(\mathrm{CN})^{2-}+\mathrm{H}^{+}$during dissociation.

If gold atoms or clusters in an acidic or near-neutral medium acquire positive charges due to protonation, then due to the excess of protons and their greater mobility, a bond is established through a proton with a complexing agent. At $\mathrm{pH}$ much higher than 7 , the protons formed due to the dissociation of water in the near-contact layer and penetrate into the electron shells of gold atoms. When protons moving away from gold, interact with hydroxyl ions, forming new water molecules. Therefore, protons, even indirectly associated with the anions of complexing agents, actively participate in the formation of bonds with gold. In addition to direct interaction with atoms of dissolved elements, clustered protons 
can also provide material-structural preparation of mineral matrices containing them. When interacting with the electron shells of metastable clusters of periodically approaching atoms of mineral-forming elements, such proton clusters form linear defects in crystal lattices, which transform into microcracks.

\section{Results and discussion}

Numerous experiments carried out by the authors of the article on the oxidation of productive mineral matrices and the leaching of chemically bound cluster gold from refractory ores, placer sands and technogenic mineral raw materials by electro-photoactivated solutions with different reagent bases confirmed the validity of the theoretical model of clustered protons [7]. Moreover, experimental facts confirming the model of cluster protonation are not only a significant increase in gold recovery when using electrophoto-activated solutions, but also a number of accompanying effects. These effects, first of all, include the abrupt nature of variations in the redox potential of leaching solution over time and a periodic increase in the redox potential of pregnant solutions.

The most illustrative comparative experiments on leaching dispersed gold were carried out at Aprelkovo mine on large-volume samples of refractory ores from the Pogromnoye deposit. Active percarbonate-cyanide solutions were prepared by processing a sodium bicarbonate solution in a photoelectrochemical reactor, followed by the introduction of sodium cyanide into them and conditioning the $\mathrm{pH}$ to 10.5 . It should be especially noted that after adding sodium cyanide to the active solution for several hours, it was not possible to determine the cyanide content in it and stabilize the $\mathrm{pH}$ (cyanide cotent detected by titration is presented on fig. 1a for standard cyanide leaching and fig. 1c for activated cyanide-sodium bicarbonate leaching). Cyanide content was detected by titration with silver nitrate. This fact most likely indicates the binding of cyanide to metastable cluster complexes by percarbonates. As a result, $70 \%$ of gold was extracted in almost 40 days using the electro-photo-activated leaching, while according to the standard scheme $-50 \%$ [8].

As can be seen from the graphs (Fig. 1), with the general nature of the change in the content of cyanides in the leaching solution, the kinetics of gold leaching when using photo-electro-activated solutions is characterized by the presence of 2 growth peaks in the gold content in pregnant solutions (Fig. 1d in comparison with 1b), which indicates the extraction of dispersed forms of gold, including clustered, starting from the 7 th day. 

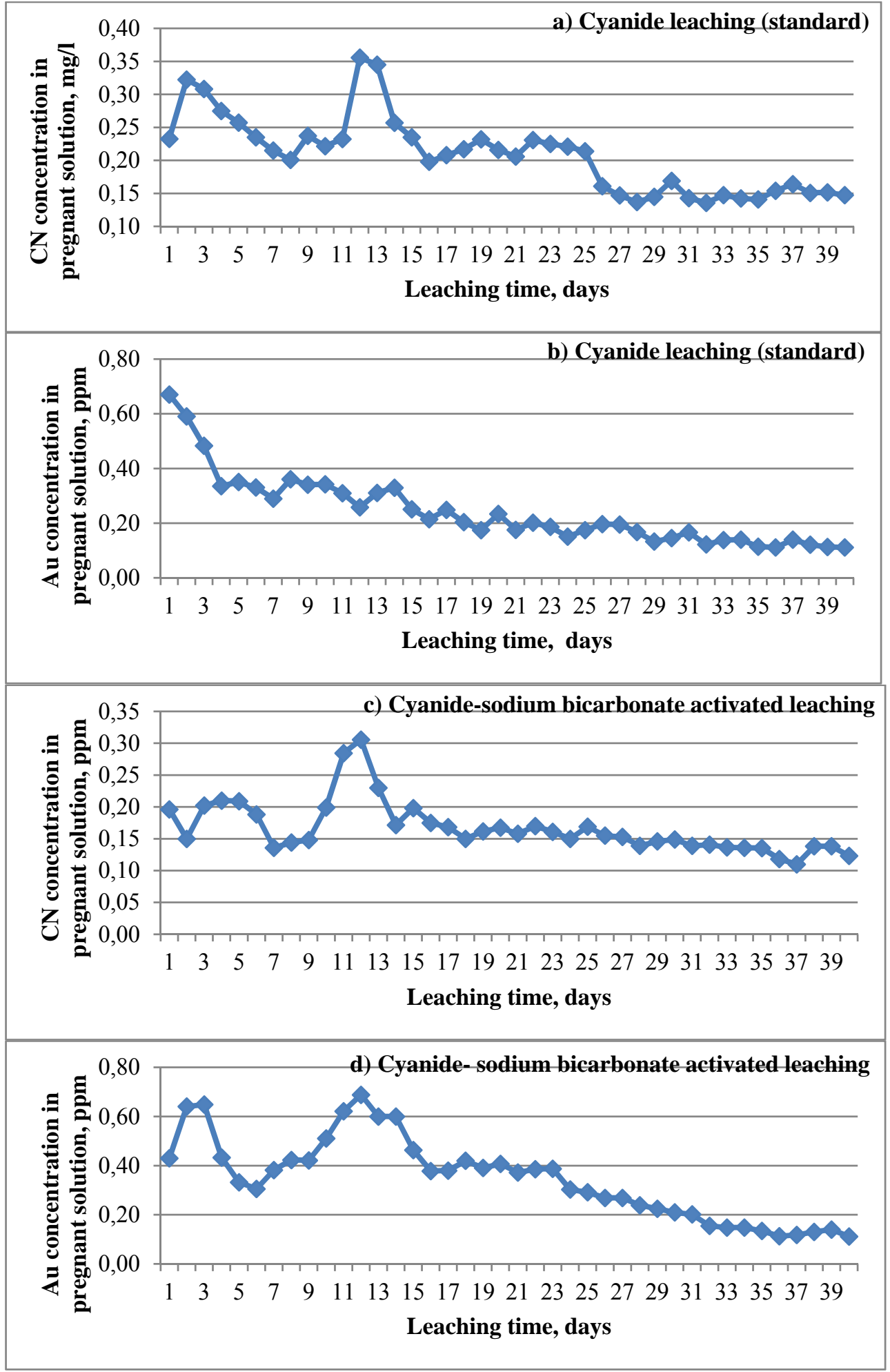

Fig. 1. Change of cyanide concentration in the pregnant solution in time and the kinetics of gold leaching according to the standard $(a, b)$ and activated (c, d) leaching 


\section{Conclusion}

The developed theoretical model of proton clustering during the electro-photo-chemical activation of leaching solutions makes it possible to explain a number of features of the kinetics and dynamics of leaching and physicochemical effects established in the course of experiments on the extraction of chemically bound cluster gold from refractory mineral raw materials. A stepwise increase in the gold content in productive solutions formed during its leaching with electro-photo-activated solutions, the abrupt nature of variations in the redox potential of leaching solutions and $\mathrm{pH}$ in time, and a periodic increase in the redox potential of pregnant solutions, indicate a significant role of protons in the system of processes that generally provide the release of chemically bound gold from crystalline lattices of host minerals and the formation of its dissolved forms.

\section{Acknowledgment}

Research was supported by scientific grant "Increasing the complexity of the use of mineral raw materials of various genesis on the basis of its systemic mineralogical and technological assessment and a differentiated approach to determining the content of precious metals by their modes of occurrence" is provided by the Government of Khabarovsk Territory (59 C, 21.08.2020).

Chemical analysis of mineral raw material and leaching pregnant solutions was carried out on the basis of the Center for Collective Use of the Khabarovsk Federal Research Center FEB RAS.

\section{References}

1. S. Helali, F. Dappozze, S. Horikoshi, T. Hoai Bui, Ch. Guillard, J. of Photochemistry and Photobiology A: Chemistry, 2551 (2013)

2. A.K. Pathak, T. Mukherjee, D. K. Maity, Chemical Physics Letters, 454, 1-310 (2008)

3. R. Prosmiti, A. García-Vela, Chemical Physics Letters, 366, 3-410 (2002)

4. Kato et al., United States Patent US 2009/0321272 A1 (2009)

5. X. Wang, L. Zhang, RSC Advances, 71 (2018)

6. A.V. Rasskazova, XXIX International Mineral Processing Congress (IMPC 2018), Moscow, Russia, September, 17-21 (2018)

7. V.N. Oparin, B.N. Smolyanitsky, A.G. Sekisov, A.I. Trubachev, V.S. Salikhov, N.V. Zykov, Journal of Mining Science, 3, 53 (2017)

8. A.G. Sekisov, A.V. Rasskazova, Patent RU No. 2647961, IPC C22B 11/00, 3/04 (2018) 